\title{
Reply to letter to the editor regarding "Use of DPP-4 inhibitors in patients with COVID-19"
}

\author{
Sebastiano Bruno Solerte ${ }^{1,2} \cdot$ Francesca D'Addio $^{3} \cdot$ Paolo Fiorina ${ }^{3,4,5}$ (i)
}

Received: 23 October 2020 / Accepted: 29 October 2020 / Published online: 17 November 2020

c) Springer-Verlag Italia S.r.l., part of Springer Nature 2020

\section{Dear Editor-in-Chief,}

We have received and read with interest the letter from Dr. Kow and Dr. Hasan [1] on our article entitled "Dipeptidyl peptidase-4 (DPP4) inhibition in COVID-19" recently published on Acta Diabetologica [2].

We all appreciate the comments and the novel information provided that complement our manuscript.

Two major points have been raised in the letter. The first one is related to the recent evidences which suggest that the development of COVID-19 is associated with episodes of thromboembolism and, in general, to a hypercoagulability state. The second one relies on the fact that the use of DPP-4 inhibitors may facilitate the establishment of a prothrombotic state in treated patients, and sitagliptin in particular has been associated with a higher probability of venous thromboembolism events. We have recently published with several international collaborators a retrospective analysis conducted on patients with type 2 diabetes and COVID-19,

Managed By Massimo Porta.

This reply refers to the article available at https://doi.org/10.1007/ s00592-020-01629-y.

Paolo Fiorina

paolo.fiorina@childrens.harvard.edu

1 Department of Internal Medicine, Geriatric and Diabetology Unit, University of Pavia, Pavia, Italy

2 School of Geriatrics, University of Pavia ASP-Pavia, Pavia, Italy

3 International Center for T1D, Pediatric Clinical Research Center Romeo ed Enrica Invernizzi, DIBIC L. Sacco, Università di Milano, Milan, Italy

4 Division of Endocrinology, ASST Fatebenefratelli-Sacco, Milan, Italy

5 Nephrology Division, Boston Children's Hospital, Harvard Medical School, 300 Longwood Ave., Boston, MA 02115, USA who, at the admission to hospital, were switched from their antidiabetic drugs to either insulin as standard-of-care treatment, or to insulin plus sitagliptin [3]. Data analyzed on 338 patients demonstrated that the addition of sitagliptin to the standard-of-care was associated with a reduced mortality and improvement of clinical outcomes. We did not observe any abnormality in prothrombotic markers; indeed, we observed a reduction in sitagliptin-treated patients of LDH, D-dimer and in general of pro-inflammatory markers, which are often associated with a hypercoagulability state. Nevertheless, we need to mention that the majority of patients with type 2 diabetes were on anticoagulant and antiplatelets medications, as a prothrombotic risk is part of diabetes as well, thus the balance between pro- and antithrombotic factors may be already altered. The letter points out also to the fact that the use of sitagliptin was associated with an increase in reporting venous thromboembolism events in diabetic patients and that this occurred in half of the patients in concomitance with an infection. The TECOS trial, however, did not demonstrate any increase in the risk of major cardiovascular events, hospitalization, death or other adverse events in patients with type 2 diabetes and established cardiovascular disease treated with sitagliptin [4]. In view of this, the use of DPP-4 inhibitors in COVID-19 may provide two major clinically relevant advantages. First, as stated by us and others [5], DPP-4 inhibitors may block the entry of SARSCoV2 into pulmonary epithelial cells due to the presence of DPP-4 as co-receptor for SARSCoV2, and second, DPP-4 inhibitors have an important anti-inflammatory effect [5], which may prevent the cytokine storm to take place and the onset of hypercoagulation phenomena. Indeed, the hypercoagulability state has been associated with a late stage of COVID-19, and it has not been clarified whether it is directly induced by a viral infection of endothelial cells or indirectly through the inflammatory and immune processes. In both cases, the use of a DPP-4 inhibitor may exert some benefits. We agree with the Authors that caution is mandatory when considering the use of DPP-4 inhibitors in 
patients with COVID-19 but without diabetes. Despite this and given the convincing results obtained in the reduction of mortality and improvement in clinical outcomes, we strongly believe that DPP-4 inhibitors, and sitagliptin among all, may represent a therapeutic option to be considered in patients with type 2 diabetes who develop COVID-19.

Author's contribution All authors contribute to the manuscript.

Funding F.D. is supported by a SID Lombardia Grant and by the EFSD/JDRF/Lilly Programme on Type 1 Diabetes Research 2019. P.F. is supported by the Italian Ministry of Health grant RF-2016-02362512 and by the Linea-2 2019 funding from Università di Milano. We thank the "Fondazione Romeo e Enrica Invernizzi" for extraordinary support.

\section{Compliance with ethical standards}

\section{Conflict of interest None.}

Ethical approval This article does not contain any studies with human participants performed by any of the authors.

Informed consent For this type of study, formal consent is not required.

\section{References}

1. Chia Siang Kow SSH. Use of DPP-4 inhibitors in patients with COVID-19. Acta Diabetol (in press)

2. Solerte SB, Di Sabatino A, Galli M, Fiorina P (2020) Dipeptidyl peptidase-4 (DPP4) inhibition in COVID-19. Acta Diabetol 57:779-783

3. Solerte SB, D'Addio F, Trevisan R, et al (2020) Sitagliptin treatment at the time of hospitalization was associated with reduced mortality in patients with type 2 diabetes and COVID-19: a multicenter, case-control, retrospective, observational study. Diabetes Care

4. Green JB, Bethel MA, Armstrong PW et al (2015) Effect of sitagliptin on cardiovascular outcomes in type 2 diabetes. $\mathrm{N}$ Engl $\mathrm{J}$ Med 373:232-242

5. Valencia I, Peiro C, Lorenzo O, Sanchez-Ferrer CF, Eckel J, Romacho T (2020) DPP4 and ACE2 in diabetes and COVID-19: therapeutic targets for cardiovascular complications? Front Pharmacol 11:1161

Publisher's Note Springer Nature remains neutral with regard to jurisdictional claims in published maps and institutional affiliations. 